\title{
Cardiovascular Pharmacology
}

National Cancer Institute

\section{Source}

National Cancer Institute. Cardiovascular Pharmacology. NCI Thesaurus. Code C18079.

Cardiovascular Pharmacology; the study of the effects of drugs upon the heart or circulatory system 